\title{
Use of the Conditional Marketing Authorization Pathway for Oncology Medicines in Europe
}

\author{
J Hoekman $^{1,2}$, WPC Boon ${ }^{1,2}$, JC Bouvy ${ }^{1}$, HC Ebbers ${ }^{1}$, JP de Jong ${ }^{3}$ and ML De Bruin ${ }^{1}$
}

Conditional marketing authorization (CMA) in the European Union (EU) is an early access pathway for medicines that show promising therapeutic effects, but for which comprehensive data are not available. Using a mixed quantitative-qualitative research design, we evaluated how CMA has been used in marketing authorization of oncology medicines in the period 2006 to 2013. We show that compared to full marketing authorization, CMA is granted based on less comprehensive data. However, this is accompanied by significantly longer assessment times and less consensus among regulators about marketing authorization. Moreover, development time from first-in-human testing to marketing authorization did not differ between full marketing authorization and CMA, but was significantly longer for CMA compared to accelerated approved products in the United States (US). Results indicate that CMA is not used by companies as a prospectively planned pathway to obtain early access, but as a "rescue option" when submitted data are not strong enough to justify full marketing authorization.

Drug regulatory agencies around the world need to strike a balance between timely access to new and needed medicines and a demand for comprehensive data on the benefits and risks of these medicines. ${ }^{1}$ The introduction of several regulatory instruments for early access to medicines since the beginning of the 1990s has provided agencies with tools to strike this balance differently, depending on the disease that is being treated. ${ }^{2}$ Early access is demanded by patients who will accept greater risks of treatment when severity of the disease is high. ${ }^{3}$ It is also supported by companies who have expressed concerns about an increasingly risk-averse regulatory environment and rising costs of drug development. ${ }^{4}$

In 2006, conditional marketing authorization (CMA) was introduced in the European Union (EU) as an early access pathway for medicines that fulfill an unmet medical need and treat seriously debilitating, life threatening, or rare diseases. The pathway allows for granting marketing authorization on the basis of "less than comprehensive clinical data" about the benefits and risks of medicines, provided that postmarketing studies are conducted to reduce uncertainties about these benefits and risks. Furthermore, CMA is granted for a renewable period of one year and products convert to full marketing authorization once all postmarketing obligations have been fulfilled and the positive benefit-risk balance has been confirmed. ${ }^{6}$

Although a substantial number of products have been granted CMA, not much is known about the clinical evidence supporting these decisions or about the process resulting in an opinion by the Committee for Medicinal Products for Human Use (CHMP) and decision by the European Commission (EC) to grant CMA. Moreover, although the CMA pathway bears many similarities with the accelerated approval pathway in the United States (US), little is known about how these two early access pathways compare.

The general purpose of this study is therefore to examine how the CMA pathway has been used in regulatory decision-making on marketing authorization of oncology medicines in the EU. We collected data on all new active substances approved for a first oncology indication by European Medicines Agency (EMA) in the period 2006 to 2013 and conducted three analyses. First, we compared products that received a full marketing authorization and CMA on evidence supporting marketing authorization, timelines from first-in-human testing to marketing authorization, and procedural characteristics of the marketing authorization decision-making process. Second, we conducted a number of interviews with company officials and regulators to obtain insights in the decision-making process resulting in the granting of CMA. Third, we compared timelines and procedural characteristics for the same oncology medicines authorized in the EU and the US. The focus on oncology medicines is motivated by the fact that these medicines constitute a relatively homogenous product group that is frequently granted CMA because of high

\footnotetext{
${ }^{1}$ Utrecht Institute for Pharmaceutical Sciences, Utrecht University, Utrecht, The Netherlands; ${ }^{2}$ Innovation Studies Group, Faculty of Geosciences, Utrecht University, Utrecht, The Netherlands; ${ }^{3}$ Exon Consultancy, Amsterdam, The Netherlands. Correspondence: J Hoekman (j.hoekman@uu.nl) 
Table 1 Characteristics of oncology medicines receiving full or conditional marketing authorization in Europe

\begin{tabular}{|c|c|c|c|}
\hline & $\begin{array}{l}\text { Full marketing authorization } \\
\qquad(n=31)\end{array}$ & $\begin{array}{l}\text { Conditional marketing authorization } \\
\qquad(n=11)\end{array}$ & $P$ value \\
\hline \multicolumn{4}{|l|}{ Pivotal trial } \\
\hline No. of patients, median [IQR] & 626 [370-808] & 154 [106-435] & $<0.001$ \\
\hline RCT, no. (\%) & $28(90)$ & $5(45)$ & 0.005 \\
\hline Blinded, no. (\%) & $11(35)$ & $2(18)$ & 0.453 \\
\hline \multicolumn{4}{|l|}{ Comparator, no. (\%) } \\
\hline Active & $15(48)$ & $1(9)$ & \\
\hline Placebo & $13(42)$ & $6(55)$ & \\
\hline No comparator & $3(10)$ & $4(36)$ & 0.006 \\
\hline \multicolumn{4}{|l|}{ Primary endpoint, no. (\%) } \\
\hline Overall survival & $19(61)$ & $0(0)$ & \\
\hline Progression-free survival & $7(23)$ & $3(27)$ & \\
\hline Time to progression & $1(3)$ & $1(9)$ & \\
\hline Response rate & $4(13)$ & $7(64)$ & $<0.001$ \\
\hline \multicolumn{4}{|l|}{ Timelines, in days, median [IQR] } \\
\hline Development time & $2,307[1,866-3,615]$ & $2,074[1,821-2,656]$ & 0.864 \\
\hline Active review time & 203 [183-210] & 204 [201-210] & 0.437 \\
\hline Clock stop time & 120 [55-159] & 190 [142-255] & 0.004 \\
\hline EC decision time & $62[57-81]$ & 84 [69-96] & 0.038 \\
\hline Total review time & $390[296-442]$ & 513 [433-569] & 0.002 \\
\hline Total development and review time & $2,692[2,155-4,029]$ & $2,507[2,312-3,244]$ & 0.829 \\
\hline Accelerated assessment, no. (\%) & $6(19)$ & $0(0)$ & 0.172 \\
\hline \multicolumn{4}{|l|}{ Procedures, no. (\%) } \\
\hline Scientific advice & $24(77)$ & $8(73)$ & 1.000 \\
\hline SAG-0 meeting & $9(29)$ & $8(73)$ & 0.029 \\
\hline List of outstanding issues & $1[1-1]$ & $1[1-2]$ & 0.063 \\
\hline Consensus vote & $27(87)$ & $6(55)$ & 0.038 \\
\hline Reexamination procedure & $1(9)$ & $0(0)$ & 0.262 \\
\hline \multicolumn{4}{|l|}{ Product characteristics, no. (\%) } \\
\hline Orphan drugs & $20(65)$ & $7(64)$ & 1.000 \\
\hline Solid cancer indication & $23(75)$ & $6(55)$ & 0.201 \\
\hline
\end{tabular}

EC, European Commission; IQR, interquartile range; RCT, randomized controlled trial; SAG-O, Scientific Advisory Group on Oncology.

Comparison testing with Wilcoxon-rank sum tests for continuous variables and Fisher's exact test for categorical variables.

unmet need. It also provides the opportunity to build on previous research that examined factors contributing to successful marketing authorization in the $\mathrm{EU}^{7}$ and qualitative differences in the authorization of oncology medicines in the US and the EU.,

\section{RESULTS}

\section{Use of CMA in the EU}

During 2006 to 2013, 44 new active substances with a first oncology indication received a positive opinion from the CHMP: 31 received full marketing authorization, 11 received CMA, and 2 received a marketing authorization under exceptional circumstances. Median number of patients in the pivotal trial was 626 (interquartile range (IQR): 370-808) for medicines receiving full marketing authorization and 154 (IQR: 106-435) for medicines receiving CMA $(P<0.001$; Table 1$)$. Although the pivotal trial supporting full marketing authorization was almost always a randomized controlled trial (90\%), the pivotal trial supporting a CMA was a randomized controlled trial (RCT) in $45 \%$ of the 
cases $(P=0.005)$. CMA products were more often authorized on the basis of response rate as primary endpoint (64\% vs. $13 \%$ ), whereas full marketing authorizations were more often based on overall survival as primary endpoint $(61 \%$ vs. $0 \% ; P<0.001)$.

Review times for products receiving CMA were significantly longer as compared with products receiving full marketing authorization. It took a median of 390 days (IQR: 296-442 days) for granting a full marketing authorization and 513 days (IQR: 433-569 days) for CMA $(P=0.002)$. The difference was due to longer clock-stop times for companies to respond to questions from CHMP and longer decision times by the EC after a positive opinion by CHMP (Table 1). We did not observe a significant difference in development times between products that were conditionally and fully authorized. Median number of days from investigational new drug (IND) submission to the marketing authorization application was 2,307 days (IQR: 1,866-3,615 days) for full marketing authorization and 2,006 days (IQR: $1,821-2,382$ days) for CMA $(P=0.864)$.

Medicines receiving full and conditional marketing authorization were equally likely to receive scientific advice from EMA on clinical development before authorization, with scientific advice being given for approximately three of four medicines in both cases $(P=1.000)$. However, conditionally authorized products were more often referred to the Scientific Advisory Group on Oncology during the marketing authorization procedure for expert recommendations on scientific and technical matters regarding the authorization decision (73\% vs. $29 \% ; P=0.029)$. Conditionally authorized products were less likely to be approved based on an unanimous positive vote (consensus vote) for authorization by all CHMP members ( $55 \%$ vs. $87 \% ; P=0.038$ ).

The results from in-depth reading of European Public Assessment Reports and semistructured interviews revealed that companies applied upfront for CMA for 2 of 11 oncology medicines that received CMA. For one product, the request for CMA was initially refused by the CHMP because of a perceived lack of "unmet medical need." As a result, regulators treated the marketing authorization application as a full marketing authorization for 10 of 11 medicines and initially applied standard benefit-risk assessment to these medicines.

Proposals to grant CMA to 10 medicines were made during the marketing authorization procedure. We found that there was one request by an applicant for CMA during an oral explanation at day 120, whereas nine proposals for CMA were made by the CHMP. Of these CHMP-initiated CMA proposals, one CMA was proposed around day 150; seven were proposed at the end of the procedure (upon or after day 180); for one medicine, CMA was proposed during a reexamination procedure.

In most cases, CMA was considered after the CHMP raised major objections regarding the possibility of granting a standard marketing authorization. In 5 of 11 cases, subsequent considerations for CMA were accompanied by restrictions of the indication to a subpopulation of patients for which a positive benefit-risk balance could be established, or to a different treatment line. Main reasons for a decision to grant a CMA included: lack of data from RCTs, lack of an RCT with an active comparator, failure to show overall therapeutic benefit in a pivotal RCT, subgroup analysis indicating that responses to treatment may differ by subpopulations, and small safety datasets.

During interviews, companies noted that they were well aware of the possibility of requesting CMA upfront, but hardly chose to use this route for oncology medicines. Three companies with five products explicitly mentioned that their regulatory strategy was to always apply for full marketing authorization, as to avoid postmarketing regulatory burden and increase chances of favorable assessment by reimbursement agencies. They also mentioned that they preferred certainty about regulatory procedures, which for them was provided by a standard marketing authorization route. For three products, companies simply expected that their data would qualify for full marketing authorization. For one product, the company mentioned a deliberate choice to file for full marketing authorization as the authorities did not provide a firm position on an upfront request for CMA during scientific advice. In addition, for two products companies mentioned that they did not adhere to scientific advice and filed early despite negative advice from regulators.

The interviewed regulators emphasized that the legislative text for both full marketing authorization and CMA explicitly mentions a "positive benefit-risk balance" as an evaluation criteria. ${ }^{6}$ They considered this necessary from a public health perspective, but noted that it blurred the distinction between evidentiary requirements for full and conditional marketing authorization. They also mentioned reluctance to grant CMA because of prior experience with lacking compliance to postmarketing studies and because they felt that legislative tools to withdraw products once they are authorized on the market are limited.

\section{Comparison of the EU and the US}

A comparison of the legislative framework for CMA and accelerated approval is provided in Table 2 . Ten of 11 conditionally authorized oncology products were also approved in the US 5 were granted a full approval, 5 accelerated approval, and one application was withdrawn by the sponsor during the procedure (Figure 1). Of 10 approved products, seven were subject to a priority review in the US compared to zero to an accelerated assessment in the EU. Furthermore, four of these products were referred to an Oncologic Drugs Advisory Committee compared to eight Scientific Advisory Group on Oncology meetings in the EU. Time to marketing authorization since IND submission was shorter in the US than in the EU for all 10 conditionally authorized products. Median difference in time from IND submission to approval was 370 days (IQR: 204-433 days) between the EU and US.

Figure 2 presents time since IND submission to approval of oncology products authorized in the EU and the US via the standard or conditional/accelerated procedure. Of 31 oncology medicines that received full marketing authorization in the EU, 28 were also approved in the US (not necessarily in the study period): 5 were granted accelerated approval and 23 full approval. In total, 14 oncology products were granted accelerated approval in the US in the period 2006-2013. Nine of these products have also been approved in Europe (follow-up until mid-2015). 
Table 2 Legislative framework for conditional marketing authorization in the EU and accelerated approval in the US

\begin{tabular}{|c|c|c|}
\hline & CMA & Accelerated approval \\
\hline Introduction & 2006 & 1992 \\
\hline Legal basis & $\begin{array}{l}\text { Commission Regulation (EC) No. } 507 / 2006 \text { of } 29 \\
\text { March } 2006 \text { falling within the scope of Regulation } \\
\text { (EC) No. } 726 / 2004 \text { of the European Parliament and } \\
\text { of the Council. }\end{array}$ & Code of Federal Regulations Part 314 Sub-Part H. \\
\hline Scope & $\begin{array}{l}\text { Seriously debilitating and life-threatening diseases; } \\
\text { medicinal products for emergency situations; orphan } \\
\text { medicinal products. }\end{array}$ & $\begin{array}{l}\text { Serious or life-threatening diseases that provide } \\
\text { meaningful therapeutic benefit to patients over exist- } \\
\text { ing treatments. }\end{array}$ \\
\hline Evaluation criteria & $\begin{array}{l}\text { Benefit-risk balance is positive; applicant is likely to } \\
\text { provide comprehensive data; unmet medical needs } \\
\text { are fulfilled; benefits of immediate availability out- } \\
\text { weigh risk. }\end{array}$ & $\begin{array}{l}\text { Medicinal product has an effect on a surrogate end- } \\
\text { point that is reasonably likely to predict clinical bene- } \\
\text { fit or an effect on a clinical endpoint other than } \\
\text { survival or irreversible morbidity. }\end{array}$ \\
\hline Postmarketing phase & $\begin{array}{l}\text { Marketing authorization is subject to postmarketing } \\
\text { obligations to provide comprehensive data; market- } \\
\text { ing authorization is valid for one year on a renewable } \\
\text { basis provided that benefit-risk balance remains } \\
\text { positive; CMA converts to standard marketing } \\
\text { authorization once postmarketing obligations are } \\
\text { fulfilled. }\end{array}$ & $\begin{array}{l}\text { Approval is subject to postmarketing commitments } \\
\text { to evaluate effect on clinical outcome; approval can } \\
\text { be subject to withdrawal if commitments are not } \\
\text { met. }\end{array}$ \\
\hline
\end{tabular}

CMA, conditional marketing authorization; EC, European Commission.

We observed a significant difference in time to marketing authorization since IND submission when comparing all 11 conditionally authorized products with all 10 accelerated approved oncology products $(P=0.003$; Table 3$)$. We did not observe a significant difference in time to marketing authorization since IND submission when comparing all fully authorized oncology products in the EU and the US $(P=0.314$; Table 3$)$.

\section{DISCUSSION}

This study examined how the CMA pathway in the EU has been used in regulatory decision-making on oncology medicines during 2006-2013. Our main findings are threefold. First, we found that CMA was granted based on less comprehensive data as compared with products receiving full marketing authorization. Yet, this did not significantly reduce the time from first-in-human testing to marketing authorization and resulted in relatively lengthy and challenging assessment procedures as compared to oncology medicines granted full marketing authorization. Second, we found that, in most cases, the granting of CMA was not proactively requested by companies, but proposed by the CHMP relatively late during the marketing authorization procedure. Third, we found that time to marketing authorization since IND submission was shorter for oncology medicines that were approved via accelerated approval in the US versus those that received CMA in the EU, but we did not observe this difference for oncology medicines that were granted a full marketing authorization in both jurisdictions.

Our results indicate that companies often apply a "wait-andsee" approach with respect to the use of CMA, whereas regulators only propose CMA after a challenging and lengthy marketing authorization procedure. The strategy of companies to not request CMA upfront results in that regulators initially apply a standard benefit-risk assessment to these medicines and only explore the possibility of CMA when they conclude that data are not comprehensive enough to grant a full marketing authorization. When this situation presents itself, regulators need to find agreement that there is still a positive benefit-risk balance, despite the fact that less comprehensive data are available. Moreover, agreement needs to be reached on whether the product meets other evaluation criteria, in particular, whether the product fills an unmet medical need and whether it is likely that comprehensive data will be provided in the near future. This situation extends marketing authorization procedures and requires increased risk tolerance on the side of the regulators resulting in a relatively lengthy process of consensus seeking as reflected by consultation of additional scientific experts during Scientific Advisory Group on Oncology meetings, requesting additional data from companies and divergent opinions among CHMP members.

The findings suggest that the use of CMA for oncology medicines is not in line with a priori policy objectives. We showed that oncology medicines with short development times in the EU, as judged from time since first-in-human testing to marketing authorization, are often granted authorization via the standard instead of conditional procedure. CMA subsequently seems to be reserved for medicines with substantial remaining uncertainties and a relative lack of consensus among CHMP members about marketing authorization. Combined with the observation that CMA is neither used as a prospectively planned strategy by companies nor for medicines with expedited development timelines, it seems that CMA is not used to bring the most promising oncology medicines earlier to the market. Rather, it has been described by companies and regulators as a "rescue option" that is used to grant marketing authorization to medicines for which there is agreement that a full marketing authorization cannot be granted. 


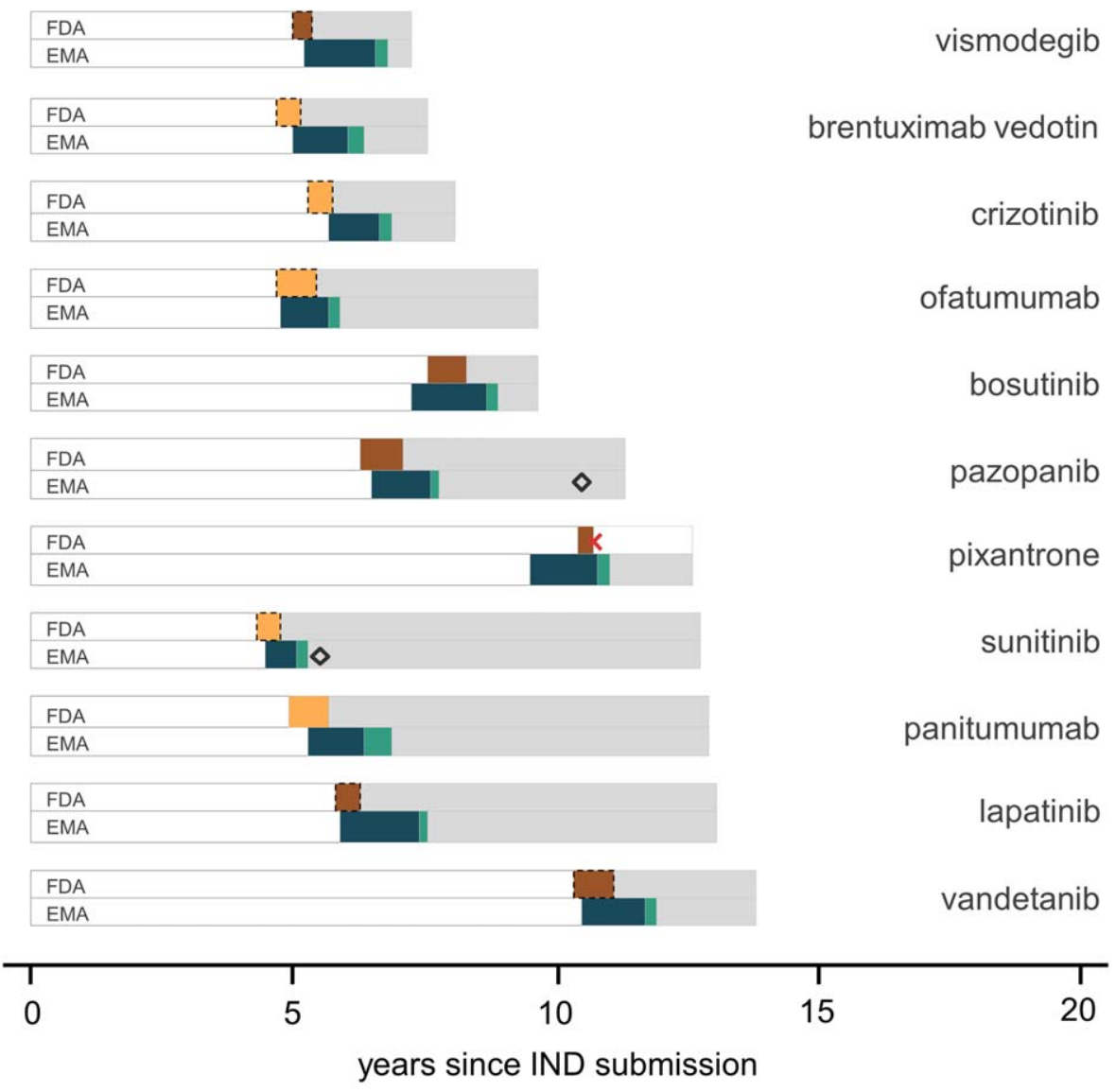

\begin{tabular}{ll}
$\square$ development time & postapproval \\
EMA approval & EC approval \\
FDA standard approval & FDA accelerated approval \\
FDA priority review & $\diamond$ CMA conversion \\
$\times$ review withdrawal & \\
\hline
\end{tabular}

Figure 1 Time to marketing authorization since IND submission in Europe and US for 11 conditionally authorized oncology products in Europe.

The study showed different approaches between the use of early-access pathways in the EU and the US. These observations may be partly driven by differences in legislation and governance structures of the two regulatory agencies. There is considerably more experience with the use of accelerated approval in the US given that this pathway was already established in 1992. Moreover, the US legislation differs because it allows for granting approval to supplemental applications in addition to initial applications. The observed overall differences in assessment times between the EU and the US, which have been well-documented previously, may also be understood in light of institutional differences between the two agencies. ${ }^{10-12}$ Furthermore, it has been found that the EMA and the US Food and Drug Administration (FDA) deal with uncertainties in different ways, with the FDA being more willing to take risks in order to provide early and quick access to new treatments. ${ }^{9}$ This study supports these obser- vations. It shows that oncology medicines authorized as accelerated approvals in the US are introduced significantly earlier on the market than those authorized via CMA in the EU, whereas this difference was not observed for full authorization by both agencies. Furthermore, our results suggest that given uncertainties about benefits and risks there is generally less need for expert feedback and prolonged assessment procedures in the US compared to the EU.

Our study complements previous empirical research on early access pathways in the EU and the US that have focused on (i) safety, (ii) compliance, and (iii) evidence supporting marketing authorization. First, studies have been motivated by a fear that early access might result in authorization of medicines that later turn out to be ineffective or unsafe. A study by Richey et al..$^{13}$ reported a higher likelihood of postapproval black box warnings to labels of oncology medicines that had received accelerated 


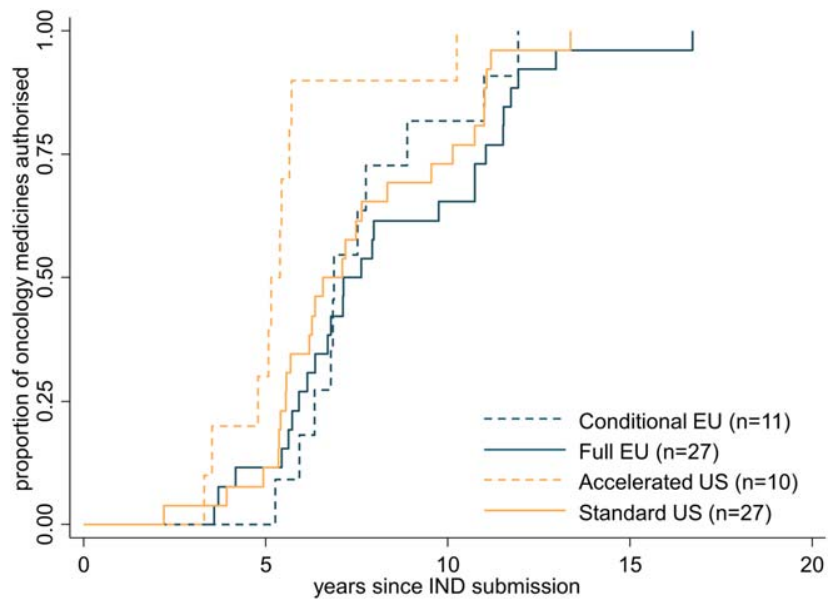

Figure 2 Time to marketing authorization of oncology medicines by pathway at the FDA or EMA.

approvals, compared with those that had received full approval. However, two European studies comparing CMA and full marketing authorization on the likelihood of safety warnings did not observe this difference. ${ }^{14,15}$ Second, studies have focused on compliance to postmarketing obligations following observations that some obligations are never completed or not started at all. For instance, a recent study on all postmarketing obligations in the US showed that of 1,781 studies requested in 2011, more than $40 \%$ had yet to start, although the total number of completed studies increased compared to an earlier cohort. ${ }^{16}$ These findings were confirmed by two other analyses. ${ }^{17,18}$ European studies on compliance have focused on postauthorization safety measures as part of pharmacovigilance activities. Results of these studies suggest that compliance is better in the EU than in the US, but could still be improved. ${ }^{19}$ Third, regarding evidence supporting marketing authorization, a recent study found that surrogate endpoints were used in almost all pivotal trials of medicines approved via the FDA accelerated approval pathway. ${ }^{20}$ Our study observed similar results with a substantial number of medicines granted CMA being approved on the base of surrogate endpoints.

The observations in this study are important for patients and healthcare professionals as well as for further development of the medicines regulatory framework in the EU. First, when a new medicine is granted CMA approval, authorization is based on less comprehensive data than is normally the case. Thus, the CMA label may be perceived by patients and healthcare professionals as a signal that the authorized medicine is promising and transfor- mative. ${ }^{21}$ However, when the CMA pathway is used as a "rescue option" for authorization, this would be a misperception about the actual therapeutic value of the medicines. Second, the CMA pathway has been put forward as the key regulatory instrument to facilitate adaptive approaches to marketing authorization in the EU. ${ }^{22}$ On the one hand, it may be expected that CMA will be used more proactively when using an adaptive pathway approach, given the prominent place for early scientific advice in this approach and the improved link with reimbursement decisions. ${ }^{23}$ On the other hand, our findings suggest that caution is necessary as the European regulatory framework seems to have difficulties to deal with high levels of uncertainty. Reflection is therefore needed on whether the observed association between a higher degree of uncertainty and more challenging procedures is rooted in legislative and guideline texts or in the mindsets and risk-perceptions of regulators and companies.

There were a number of limitations to this study. First, our research was limited to the oncology setting and it is unclear whether the results of this study can be extrapolated to other indication settings, such as human immunodeficiency virus, tuberculosis, epilepsy, or cystic fibrosis. CMA procedures for these latter indications may differ because of differences in rarity of the disease, perceived unmet medical need, availability of alternative treatment options, or differences in the level of scientific knowledge. Second, there may be other differences between oncology medicines receiving CMA and full marketing authorization that may explain some of the observed patterns in the study. We did, however, not observe such differences (e.g., by cancer indication or orphan status). Third, because of data limitations we only focused on products that obtained a marketing authorization and did not consider products that received a negative opinion. Hence, we do not know to what extent CMA is considered by companies and regulators for products that ultimately received a negative opinion from the CHMP.

In conclusion, we show that actual use of CMA for oncology medicines deviates from a priori policy objectives. CMA is not used by companies as a prospectively planned pathway, but rather seems to provide a way out for companies and regulators when data are not strong enough to justify a full marketing authorization. It follows that use of CMA for oncology medicines does not seem to be reserved for the most promising and transformative medicines. This unintended use bears a risk of false-positive regulatory decision-making and is not well aligned with expectations of patients and healthcare professionals about the function of early access pathways.

Table 3 Time to marketing authorization since IND submission in Europe and the US

\begin{tabular}{llll}
\hline \multicolumn{1}{l}{ EU } & & US & \\
\hline Time to authorization since IND, median [IQR] & & & \\
\hline Full authorization & $2,692[2,155-4,029]$ & $2,495[2,024-3,696]$ & 0.314 \\
\hline Conditional (EU)/accelerated (US) & $2,507[2,312-3,244]$ & $1,923[1,746-2,063]$ & 0.003
\end{tabular}

EU, European Union; IND, investigational new drug; US, United States.

Median [IQRs] are reported. Comparison testing with Wilcoxon-rank sum tests. 


\section{METHODS}

This study used a mixed quantitative-qualitative research design combining a cohort analysis of oncology medicines with semistructured interviews with companies and regulators.

\section{Cohort analysis}

We collected information on all initial marketing authorizations of new active substances that were granted a first oncology indication by the EMA in the period 1 January 2006 to 31 December 2013. The list of oncology medicines was compiled through the Agency's website and triangulated with published sources. ${ }^{8,24,25}$ We excluded medicines for supportive or palliative care, generics, biosimilars, and new formulations or dosing regimens of already authorized medicines. As a result, one oncology medicine with a CMA (Votubia, Everolimus) that was already approved for another indication (Afinitor, Everolimus) was excluded from our cohort.

We extracted information on product characteristics, evidence submitted for marketing authorization, review timelines, and regulatory procedures before (scientific advice) and during the assessment (scientific advisory group meeting, consensus vote, number of outstanding issues) from European Public Assessment Reports and EMA Annual Reports. In addition, we determined whether the medicines were approved by the FDA based on information from the Drugs@FDA.gov website and extracted data regarding review timelines, regulatory decisions on marketing authorization, and date of submission of the IND application (which marks the start of first-in-human testing). Dates of IND submission were missing for six medicines of which four were not authorized in the US. All data were collected independently by two investigators (J.H. and W.B. or J.H. and J.B.) and disagreement was resolved by consensus.

We compared characteristics of medicines and marketing authorization procedures that resulted in the granting of a CMA and a full marketing authorization by means of Wilcoxon rank-sum tests for continuous data and Fisher's exact tests for categorical variables. Development timelines were computed by the number of months between the date of IND submission and the date of acceptance of the marketing authorization application in the EU or the US. As no centralized decision on first-in-human testing is made in the EU, we used time since submission of IND for the calculation of European development times.

\section{Interviews}

We conducted eight semistructured interviews with six sponsors in order to obtain insight in the decision-making process resulting in the granting of CMA. The interviews were conducted with regulatory affairs representatives of large pharmaceutical companies with multiple authorized medicines in the EU. The interviews covered marketing authorization procedures of 10 of 11 conditionally authorized medicines in our sample. Interview questions focused on the motivations of companies to prospectively consider a request for CMA, as well as on events during the marketing authorization procedure that were key to the final decision to grant a CMA.

We also conducted three semistructured interviews with (former) regulators and EC officials with substantial involvement in the design and/ or use of the CMA procedure. These interviews aimed to elucidate motivations for creating the CMA pathway and to obtain a general understanding of the implementation of the CMA pathway so far. In addition, early results on the use of the CMA procedure were presented at several multi-stakeholder workshops where regulators, companies, payers, and patient representatives were invited to comment on findings.

Interviews were conducted by two investigators (J.H. and W.B.) and prepared on the base of in-depth reading of the European Public Assessment Reports and policy documents pertaining to CMA. The semistructured interviews consisted of a number of relatively open questions. To ensure that interview respondents covered relevant topics while answering these questions, the interviewers used a list of discussion issues to prompt further questioning. Questions and discussion issues asked to companies and regulators are included in Appendix I. All interviews were transcribed in full by one investigator (J.H.) and reviewed by a second investigator (W.B.). All interviews were coded on motivations and considerations for using CMA. Interviews with sponsors that covered individual procedures were tabulated for moments when CMA was (first) discussed internally and with regulators. Analyses were conducted with Nvivo QSR 10.

\section{ACKNOWLEDGMENTS}

This research has been performed within the context of "Escher Projects" that resides under the umbrella of Dutch public-private partnership Top Institute Pharma (www.ti-pharma.com) and has received funding from European Federation of Pharmaceutical Industry \& Associations (EFPIA) and the Association of the European SelfMedication Industry (AESGP). We gratefully acknowledge all interview respondents for their contribution as well as the valuable discussions about the results with participants at the Escher Workshop organized in Utrecht on 13 May 2014 and the DIA/TOPRA Meeting on 18 September 2014. Part of this research has been published in the report: "Improving the EU system for marketing authorisation: Learning from regulatory practice" that can be downloaded from: http://escher.tipharma.com/. The research has also been presented at the International Conference on Pharmacoepidemiology and Therapeutic Risk Management, Taiwan, 2014 and at the first meeting of the European Commission Expert Group on Safe and Timely Access to Medicines for Patients, Brussels, 2015.

\section{AUTHOR CONTRIBUTIONS}

J.H., W.B., J.C.B., H.C.E., J.P.D.J., and M.L.D.B. wrote the manuscript; J.H., W.B., J.C.B., H.C.E., J.P.D.J., and M.L.D.B. designed the research; J.H., W.B., and J.C.B. performed the research; J.H. and M.L.D.B. analyzed the data.

(c) 2015 American Society for Clinical Pharmacology and Therapeutics

1. Eichler, H.G., Pignatti, F., Flamion, B., Leufkens, H. \& Breckenridge, A. Balancing early market access to new drugs with the need for benefit/risk data: a mounting dilemma. Nat. Rev. Drug Discov. 7, 818-826 (2008).

2. Baird, L.G. et al. Accelerated access to innovative medicines for patients in need. Clin. Pharmacol. Ther. 96, 559-571 (2014).

3. Eichler, H.G. et al. The risks of risk aversion in drug regulation. Nat. Rev. Drug Discov. 12, 907-916 (2013).

4. Scannell, J.W., Blanckley, A., Boldon, H. \& Warrington, B. Diagnosing the decline in pharmaceutical R\&D efficiency. Nat. Rev. Drug Discov. 11, 191-200 (2012).

5. European Medicines Agency. Guideline on the scientific application and the practical arrangements necessary to implement Commission Regulation (EC) No. 507/2006 on the conditional marketing authorisation for medicinal products for human use falling within the scope of regulation (EC) No 726/2004. Doc. Ref. EMEA/509951/ 2006. <http://www.ema.europa.eu/docs/en_GB/document library/Scientific_guideline/2009/10/WC500004908.pdf> (2006). Accessed 20 February 2015.

6. European Commission. Commission Regulation (EC) No 507/2006 of 29 March 2006 on the conditional marketing authorisation for medicinal products for human use falling within the scope of Regulation (EC) No 726/2004 of the European Parliament and of the Council. Official Journal of the European Union 2006; L/92:6-9. $<$ http://ec.europa.eu/health/files/eudralex/vol-1/reg_2006_507/ reg_2006_507_en.pdf > (2006). Accessed 20 February 2015.

7. Putzeist, $\mathbf{M}$. et al. Factors influencing non-approval of new drugs in Europe. Nat. Rev. Drug Discov. 11, 903-904 (2012).

8. Trotta, F., Leufkens, H.G., Schellens, J.H., Laing, R. \& Tafuri, G. Evaluation of oncology drugs at the European Medicines Agency and US Food and Drug Administration: when differences have an impact on clinical practice. J. Clin. Oncol. 29, 2266-2272 (2011). 
9. Tafuri, G. et al. How do the EMA and FDA decide which anticancer drugs make it to the market? A comparative qualitative study on decision makers' views. Ann. Oncol. 25, 265-269 (2014).

10. Downing, N.S., Aminawung, J.A., Shah, N.D., Braunstein, J.B., Krumholz, H.M. \& Ross, J.S. Regulatory review of novel therapeutics - comparison of three regulatory agencies. N. Engl. J. Med. 366, 2284-2293 (2012).

11. Roberts, S.A., Allen, J.D. \& Sigal, E.V. Despite criticism of the FDA review process, new cancer drugs reach patients sooner in the United States than in Europe. Health Aff. (Millwood) 30, 1375-1381 (2011).

12. Shah, R.R., Roberts, S.A. \& Shah, D.R. A fresh perspective on comparing the FDA and the CHMP/EMA: approval of antineoplastic tyrosine kinase inhibitors. Br. J. Clin. Pharmacol. 76, 396-411 (2013).

13. Richey, E.A. et al. Accelerated approval of cancer drugs: improved access to therapeutic breakthroughs or early release of unsafe and ineffective drugs? J. Clin. Oncol. 27, 4398-4405 (2009).

14. Boon, W.P., Moors, E.H., Meijer, A. \& Schellekens, H. Conditional approval and approval under exceptional circumstances as regulatory instruments for stimulating responsible drug innovation in Europe. Clin. Pharmacol. Ther. 88, 848-853 (2010).

15. Arnardottir, A.H., Haaijer-Ruskamp, F.M., Straus, S.M., Eichler, H.G., de Graeff, P.A. \& Mol, P.G. Additional safety risk to exceptionally approved drugs in Europe? Br. J. Clin. Pharmacol. 72, 490-499 (2011).

16. Fain, K., Daubresse, M. \& Alexander G.C. The Food and Drug Administration Amendments Act and postmarketing commitments. JAMA 310, 202-204 (2013).
17. Moore, T.J. \& Furberg, C.D. Development times, clinical testing, postmarket follow-up, and safety risks for the new drugs approved by the US Food and Drug Administration: the class of 2008. JAMA Intern. Med. 174, 90-95 (2014).

18. Willyard, C. FDA's post-approval studies continue to suffer delays and setbacks. Nat. Med. 20, 1224-1225 (2014).

19. Blake, K.V. et al. European Medicines Agency review of postauthorisation studies with implications for the European Network of Centres for Pharmacoepidemiology and Pharmacovigilance. Pharmacoepidemiol. Drug Saf. 20, 1021-1029 (2011).

20. Downing, N.S., Aminawung, J.A., Shah, N.D., Krumholz, H.M. \& Ross, J.S. Clinical trial evidence supporting FDA approval of novel therapeutic agents, 2005-2012. JAMA 311, 368-377 (2014).

21. Kesselheim, A.S. \& Darrow, J.J. FDA designations for therapeutics and their impact on drug development and regulatory review outcomes. Clin. Pharmacol. Ther. 97, 29-36 (2015).

22. Eichler, H.G. et al. Adaptive licensing: taking the next step in the evolution of drug approval. Clin. Pharmacol. Ther. 91, 426-437 (2012).

23. Eichler, H.G. et al. From adaptive licensing to adaptive pathways: delivering a flexible life-span approach to bring new drugs to patients. Clin. Pharmacol. Ther. 97, 234-246 (2014).

24. Shea, M.B., Roberts, S.A., Walrath, J.C., Allen, J.D. \& Sigal, E.V. Use of multiple endpoints and approval paths depicts a decade of FDA oncology drug approvals. Clin. Cancer Res. 19, 3722-3731 (2013).

25. Munos, B. A forensic analysis of drug targets from 2000 through 2012. Clin. Pharmacol. Ther. 94, 407-411 (2013). 